\title{
High spectral efficiency coherent superchannel transmission with soliton microcombs
}

\author{
Mikael Mazur* Student Member OSA, IEEE, Myoung-Gyun Suh* Member OSA, IEEE, Attila Fülöp, Jochen \\ Schröder Senior Member OSA, Member IEEE, Victor Torres-Company Senior Member OSA, Magnus \\ Karlsson Fellow OSA, Senior Member IEEE, Kerry J. Vahala Fellow OSA, IEEE and Peter A. \\ Andrekson Fellow OSA, IEEE \\ *These authors contributed equally to this work
}

\begin{abstract}
Spectral efficiency (SE) is one of the key metrics for optical communication networks. An important building block for its maximization are optical superchannels, channels that are composed of several subchannels with an aggregate bandwidth larger than the bandwidth of the detector electronics. Superchannels which are routed through the network as a single entity, together with flex-grid routing, allow to more efficiently utilize available bandwidth and eliminate the guardbands between channels, thus increasing spectral efficiency. In contrast to traditional wavelength division multiplexing (WDM) channels, subchannel spacing and thus superchannel SE is governed by the linewidth and stability of the frequency spacing of the transmitter lasers. Integrated optical frequency combs, particulary the parametrically generated so-called microcombs, which provide optical lines on a fixed frequency grid are a promising solution for low power superchannel laser sources that allow to minimize the SE loss from suboptimal channel spacing. However, it is extremely challenging to realize micro-combs with sufficient line power, coherence and line spacing that is compatible with electronic bandwidths. Because the line-spacing generated by most devices is above $40 \mathrm{GHz}$, demonstrations often rely on additional electro-optic frequency shifter or divider stages to avoid digital-to-analog-converter (DAC) performance degradation when operating at high symbol rates. Here we demonstrate a 50 -line superchannel from a single $22 \mathrm{GHz}$ line spacing soliton microcomb. We demonstrate $12 \mathrm{~Tb} / \mathrm{s}$ throughput with $>10 \mathrm{bits} / \mathrm{s} / \mathrm{Hz}$ SE efficiency after $80 \mathrm{~km}$ transmission and $8 \mathrm{~Tb} / \mathrm{s}$ throughput $(\mathrm{SE}>6 \mathrm{bits} / \mathrm{s} / \mathrm{Hz}$ ) after $2100 \mathrm{~km}$, proving the feasibility and benefits of generating high signal quality, broadband waveforms directly from the output of a micro-scale device with a symbol rate close to the comb repetition rate.
\end{abstract}

\section{INTRODUCTION}

Superchannels are broadband (typically significantly larger than the bandwidth of the detecting electronics) optical chan-

M. Mazur was with the Photonics Laboratory, Fibre Optic Communication Research Centre (FORCE), Department of Microtechnology and Nanoscience, Chalmers University of Technology, Gothenburg SE-412 96, Sweden. He is now with Nokia Bell Labs, Crawford Hill Laboratory, Holmdel, NJ 07733, USA. M.G. Suh was with the T. J. Watson Laboratory of Applied Physics, California Institute of Technology, Pasadena, CA 91125, USA. He is now with the Physics \& Informatics Laboratories, NTT Research A. Fülöp was with the Photonics Laboratory, Fibre Optic Communication Research Centre (FORCE), Department of Microtechnology and Nanoscience, Chalmers University of Technology, Gothenburg SE-412 96, Sweden. He is now with (now Nvidia), Hugo Grauers Gata 3B, SE-411 33 Göteborg, Sweden. J. Schröder, V. Torres-Company, M. Karlsson and P. A. Andrekson are with the Photonics Laboratory, Fibre Optic Communication Research Centre (FORCE), Department of Microtechnology and Nanoscience, Chalmers University of Technology, Gothenburg SE-412 96, Sweden K. Vahala is with the T. J. Watson Laboratory of Applied Physics, California Institute of Technology, Pasadena, CA 91125. USA. nels composed of densely spaced, WDM subchannels that are routed through the network as a single unit (Fig. 1). With no filtering or routing guardbands needed within the channel, the channel spacing can be significantly reduced thereby improving the spectral efficiency (SE) [1]. Integrated, multiwavelength transceivers have enabled high-rate superchannels with reduced foot-print and energy dissipation [2]. However, integration itself does not improve the system SE. When forming a superchannel using typical independent lasers, the minimum required guardband is limited by laser frequency uncertainties to a few $\mathrm{GHz}$ [3], [4]. This limitation leads to a loss in efficiency of about $10 \%$ for sub-channel symbol rates of around 30 Gbaud [4]. The guardband penalty is therefore the strongest factor limiting SE and thus system data rates. High symbol rates help to mitigate this problem, but at the expense of increasing the penalty associated with DAC and analog-todigital converter (ADC) imperfections [5]. To fully overcome this problem requires to use frequency-locked carriers instead.

Optical frequency combs, sources of optical lines with a fixed frequency spacing, intrinsically provide frequency locked carriers and have therefore been a crucial element in recent high SE demonstrations [6], [7], [8]. Beyond maximizing SE, frequency locking between the lines of a frequency comb can also enable novel DSP concepts to improve performance [9], [10] and/or reduce energy consumption [11].

In addition, to reach high spectral efficiencies high-order modulation formats are needed, e.g., 16,64 or 256-QAM. However, these require high signal-to-noise ratios (SNRs) and small phase noise. From a source perspective, it is the channel power and linewidths that determines these properties, and it remains to be proven that integrated frequency combs can meet the requirements of also these higher order formats over broad bandwidths and long transmission distances [11], [12].

Amongst the different comb generation techniques, demonstrations of comb formation in micro-cavities, so-called soliton microcombs, have presented a major turning point for ubiquitous comb applications (e.g. frequency synthesis[13], spectroscopy [14], [15], laser-ranging [16], [17] and optical clocks [18]) because of their compact form factor and low operating power [19], [20]. Moreover, soliton formation ensures highly stable mode locking and reproducible spectral envelopes[21], [22]. These properties make them attractive for superchannel generation in optical communication. However, the highest superchannel SE so far have been demonstrated 


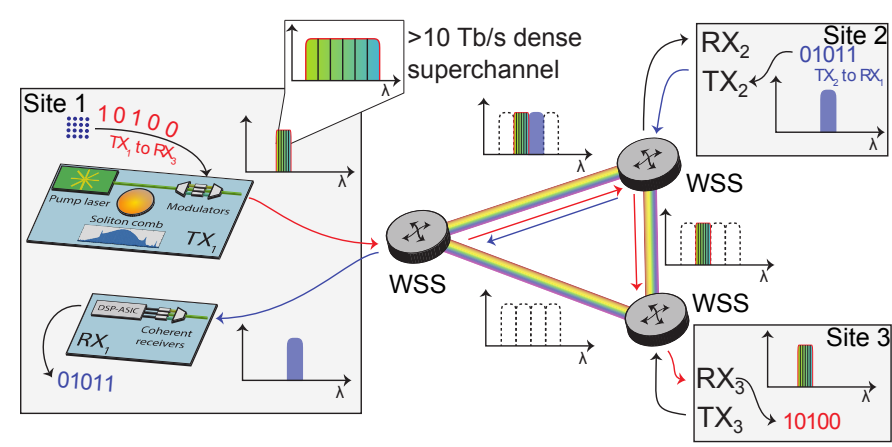

Fig. 1. Concept of microcomb-based superchannels in future networks. In an all-optically routed network, superchannels can be routed arbitrarily across the entire system. They enable multi- $\mathrm{Tb} / \mathrm{s}$ throughput with high spectral efficiency by avoiding excising guardbands between channels which are needed to separate individual channels using wavelength selective switches (WSS). The sketch shows an example of such a scenario using a chip-scale microcombbased superchannel transmitter (Tx). The superchannel is transmitted from site 1 to a receiver $(\mathrm{Rx})$ in site 3 (red) while a second superchannel goes from site 2 to site 1 (blue). High spectral line density microcombs allow the superchannels to be generated on a chip while maintaining state-of-the-art data spectral effiencies, thereby maximizing the throughput of the entire network.

using discrete electro-optic combs [6], [7], because most chipintegrated comb solutions exhibit combs with relatively high line-spacing (typically $>50-100 \mathrm{GHz}$ ) which cannot easily be matched by DACs and ADCs [5]. Microcomb transmissions experiments have therefore focused on demonstrating transmission with large bandwidths and used interleaving of two combs to reach $50 \mathrm{~Tb} / \mathrm{s}$ at a modest $\mathrm{SE}$ of $5.2 \mathrm{bits} / \mathrm{s} / \mathrm{Hz}$ [23]. Instead networks in the mid-term rather require modest bandwidth, but high SE superchannels. To overcome the limitations of integrated combs, researchers have resorted to additional electro-optic frequency shifters [24], or used single-sideband modulation [25] to avoid the need for operating at high symbol rates. Additional frequency-shifters would significantly increase integration complexity and introduce additional losses that would be hard to compensate on a fully integrated transmitter. The additional integration complexity of ref. [25] is minor in comparison (one additional splitter per channel), however it requires a sufficiently high DAC analog bandwidth, which could instead be used to reduce channel count and thus components. The highest SE from a single microcomb device modulating a single channel per comb line has been $6.7 \mathrm{bits} / \mathrm{s} / \mathrm{Hz}$ using a non-soliton microcomb [26], significantly below demonstrations with electro-optic frequency generation.

Here we demonstrate a microcomb-based superchannel transmitter with state-of-the-art spectral efficiency that has previously only been possible by generating additional frequency components electro-optically [24]. Compared to previous experiments in optical communications[27], [23], [28], [29], [25], our microcomb features a narrow line spacing of $22.1 \mathrm{GHz}$, based on a high optical Q-factor whisperinggallery-mode silica resonator [30]. The high Q significantly reduces the parametric oscillation threshold power, while simultaneously providing a four orders of magnitude more stable line spacing compared to free-running lasers [3]. We demonstrate transmission of a $12 \mathrm{~Tb} / \mathrm{s}$ superchannel over distances ranging from a single $82 \mathrm{~km}$ span with a SE exceeding

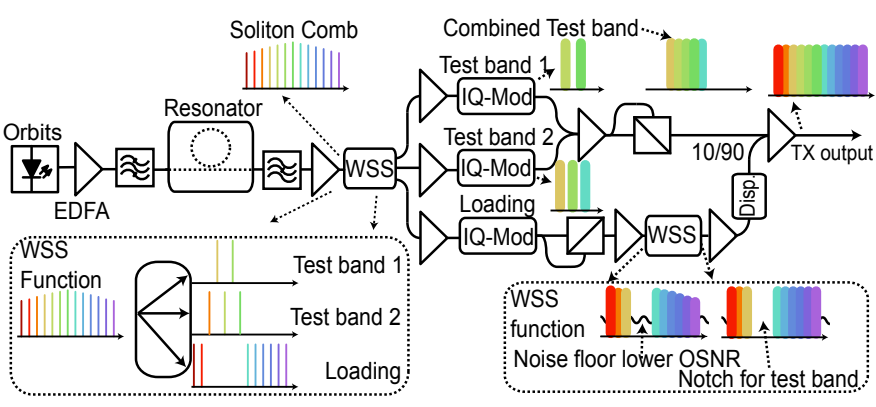

Fig. 2. Detailed transmitter setup to generate the superchannel. EDFA: Erbium-doped fiber amplifier, WSS: Wavelength Selective Switch (Waveshaper), IQ-Mod: Intensity-Phase modulator, Disp: Dispersion.

$10 \mathrm{bits} / \mathrm{s} / \mathrm{Hz}$, to $2000 \mathrm{~km}$ with a SE higher than $6 \mathrm{bits} / \mathrm{s} / \mathrm{Hz}$. Our superchannel features a reduction in the required guardband by two orders of magnitude compared to previous demonstrations using a single soliton microcomb without electrooptic frequency generation [23]. This enables sub-GHz interchannel guardbands. Combined with further advances in hybrid integration [31], high-SE microcomb-based transmitters can therefore enable novel transmission schemes with lower energy consumption [11] while unlocking continued growth in optical communications capacity. This article significantly extends our previous conference demonstration [32] with detailed characterisation of the performance and the trade-offs being made in comparison to single laser transmission.

\section{EXPERIMENT}

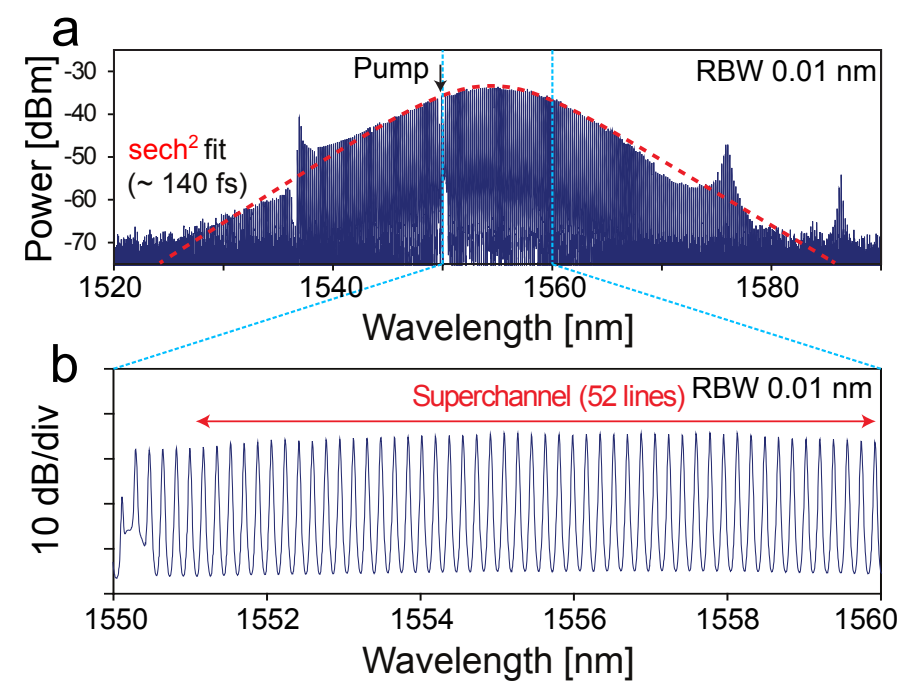

Fig. 3. Optical spectrum of the micro-comb: a) full spectrum, b) lines used for the superchannel transmission experiment.

To demonstrate the feasibility of high SE microcomb-based superchannels we conducted two transmission experiments. The experimental setup of the transmitter is shown in Fig. 2. Light from a $1550 \mathrm{~nm}$ fiber laser (Ethernal SlowLight Laser manufactured by Orbits Lightwave, Inc.) was amplified to approximately $150 \mathrm{~mW}$ by an EDFA and coupled to the packaged high-Q (> 800 million) silica microresonator with $3 \mathrm{~mm}$ diameter corresponding to $22.1 \mathrm{GHz}$ FSR (For the device 
a

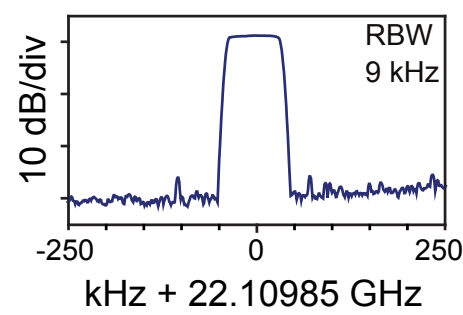

b

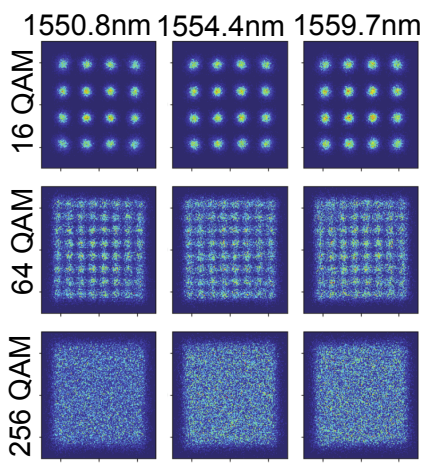

Fig. 4. a) Electrical spectrum of the comb-repetition rate over several hours. b) Constellation diagrams of selected channels for different modulation formats after $82 \mathrm{~km}$ of transmission (x-polarization).

fabrication process and typical performance characteristics see [30], [33]). The low short-term linewidth $(<1 \mathrm{kHz})$ fiber laser was used due to its improved robustness against external vibrations and acoustic noise. The device was packaged in a compact module for better thermal stability and portability. The soliton is generated via frequency tuning of the pump laser and fast stopping inside the soliton existence detuning range via piezo control (see [34] for more details on relevant parameters). The spectrum of the generated stable soliton micro-comb is shown in Fig. 3a. While the comb was pumped at $1549.9 \mathrm{~nm}$, the Raman self-frequency shift[35], [36], [37] displaces the spectral center of the soliton envelope to near $1555 \mathrm{~nm}$. This enables selection of 52 carriers around $1555 \mathrm{~nm}$ without the need to sacrifice any carriers around the pump notch filter, which is a crucial aspect to maximizing the system efficiency. The zoomed-in optical spectrum in Fig. 3b shows the equidistantly-spaced comb lines over $10 \mathrm{~nm}$ span measured after amplification, which were used for the transmission experiment. The electrical spectrum (Fig. 4a) shows that the soliton repetition rate of $22.1 \mathrm{GHz}$ is stable within a range of $100 \mathrm{kHz}$ over several hours.

The device temperature was controlled via a TEC element installed inside the module, while a fast feedback loop maintained the pump laser frequency [38], enabling stable soliton operation (absolute frequency stability better than $200 \mathrm{kHz}$ at $100 \mathrm{~s}$ averaging) over the duration of the entire transmission experiment ( $>100$ hours). The generated comb was passed through a notch filter to suppress the residual pump and directly amplified using an EDFA with about $5 \mathrm{~dB}$ of noise figure. For signal modulation we followed the common testloading-band approach to ensure realistic carrier optical signalto-noise ratio (OSNR) and minimize any penalty from not having independent modulators for each channel [39]. For this a Wavelength Selective Switch (WSS) separated the lines into 3 paths; the loading band, and the 5 channel test-band with separate paths for odd and even modes. The per-line power of the test-band channels was approximately $10 \mathrm{dBm}$ into the modulator. The two test-band IQ-modulators were driven by 2 independent DACs (Keysight M8195A), operating at $60 \mathrm{Gs} / \mathrm{s}$ to generate 21.5 Gbaud $M$-ary quadature amplitude modulation channels ( $M$-QAM with $M \in[16,64,256])$ and a rootraised cosine (RRC) pulse-shape with $1 \%$ roll-off. The symbol rate was optimized to maximize $\mathrm{SE}$ by balancing guardband overhead and linear cross-talk. After modulation the test-band channels were combined and polarization multiplexed using the split-delay-combine method (delay $\approx 250$ symbols). The remaining channels in the loading band were modulated using a single modulator and polarization multiplexed and optically dispersed to emulate independent data on each channel. An additional WSS was used to surpress amplified spontaneous emission (ASE) noise in the notch for the channel testband. Test- and loading-band were then combined and amplified before coupling to the transmission link. The link consisted of either a single span of $82 \mathrm{~km}$ of standard single-mode fiber (SSMF) or two consecutive spans placed inside a recirculating loop. The span-loss was about $16.5 \mathrm{~dB}$ for each span which was compensated using EDFAs with about $5.5 \mathrm{~dB}$ noise figure. A WSS and a $10 \mathrm{~nm}$ bandpass filter inside the recirculating loop ensured a flat spectrum and prevented the build up of out-of-band amplifier noise. A polarization scrambler was used to randomly vary the state-of-polarization into the first span of the loop. We found an optimized launch power for both single span and loop transmission of $13.5 \mathrm{dBm}$. The receiver consisted of a pre-amplifier followed by a $0.25 \mathrm{~nm}$ bandpass channel selection filter and a standard coherent receiver with $23 \mathrm{GHz}$ analogue bandwidth using a free-running external cavity laser (linewidth $\approx 100 \mathrm{kHz}$ ) as local oscillator (LO). The electrical signal was digitized with a $50 \mathrm{GS} / \mathrm{s}$ real-time oscilloscope and processed offline with a pilot-aided DSP [40] (available open-source [41]), with optimized pilot overheads of $1.5 \%$ and $2.6 \%$ for single and loop measurements respectively. No data-aided pre-convergence or adaptation using the payload symbols was performed. Similar to [40], we optimized the pilot insertion ratio to maximize the system throughput. The maximum frame length was limited by the available DAC memory to 93440 symbols and we found that a pilot sequence length of 1024 symbols, corresponding to about $1 \%$ overhead, resulted in the highest throughput for single span transmission. For long-haul, this was expanded to 2048 symbols to enhance the tolerance to transmission impairments. The insertion ratio for phase tracking pilots was 1 pilot per 256 symbols, corresponding to about $0.4 \%$ overhead, in both cases. For the longhaul measurements using PM-16QAM, a blind carrier phase recovery algorithm [42] was used in addition to the pilotbased algorithm. Note that while completely blind processing should result in the lowest overhead, performance is strongly limited by cycle slips resulting in large variations between batches. The use of pilots avoided this and a much smaller tracking range for the blind phase search can be used, reducing implementation complexity and power consumption [43].

Signal performace was characterized in terms of bit error rate (BER) (assuming a pre soft-decision forward error correction (FEC) BER threshold of $4 \cdot 10^{-2}$ for $20 \%$ FEC data overhead [44]) and generalized mutual information (GMI), which is a measure of potential throughput given an ideal bitwise FEC code [45]. BER and GMI were calcuated based on $>10$ million bits per wavelength channel in three measurement batches. The SE is calculated from the GMI by deducting over- 
heads from the guardbands (2.7\%) and DSP pilots, resulting in a GMI-based achievable information rate (AIR).

\section{RESULTS}

We optimized and characterized the system with several experiments. First we investigate the optimum modulation format and symbol rate by performing modulation order sweeps using a single carrier and symbol rate sweeps for multiple carriers in a back-to-back (B2B) configuration. The transceiver penalties are then characterized in B2B noise loading experiments before performing single- and multi-span transmission experiments.

\section{A. Transmission Optimization}

To maximize the spectral efficiency, modulation order needs to be optimized with respect to limited signal-to-noise ratio provided by the transceiver electronics. We therefore swept the modulation order for single carrier and measured the resulting performance using both GMI and BER, as shown in Fig. 5. We also observe a penalty of about $0.3 \mathrm{bit} / 4 \mathrm{D}$-symbol for PM-64QAM transmission with respect to the maximum information content of 12 bits/4D-symbol. The highest performance is at PM-256QAM reaching 12.7 bits/4D symbol. For higher modulation formats performance degrades, which we note is unexpected assuming Gaussian noise. We attribute this effect to nonlinear transceiver impairments which effect higher modulation formats more strongly.

In addition the very dense packing required to form an efficient superchannel results in a penalty due to linear crosstalk between neighboring channels. This is shown in Fig. 5 where the channel symbol rate is varied to maximize the resulting spectral efficiency. We observe that for both 64QAM and 256QAM, the optimal baud-rate is 21.5 Gbaud and this was chosen for the transmission experiment. The spectral overhead from the $\sim 600 \mathrm{MHz}$ guard band was $2.7 \%$.

\section{B. Noise loading}

To verify the performance of our transmitter, we performed noise loading measurements. The results for PM-16QAM and PM-64QAM are shown in Fig. 6. We compared the case of directly using the transmitter laser with comb lines corresponding to the lower, upper and center wavelength used to form the superchannel. The implementation penalty is quantified with respect to theory at the target threshold of $4 \cdot 10^{-2}$ [44]. For 21.5 Gbaud PM-64QAM, we observe an implementation penalty of about $1 \mathrm{~dB}$ with respect to theory when only using the comb seed laser. In addition, the penalty from using the frequency comb ranges from $<0.5 \mathrm{~dB}$ to about $1.5 \mathrm{~dB}$. The highest penalty is observed for the lowest wavelength line, being about $0.5 \mathrm{~dB}$ higher than the highest used wavelength. This was due to varying gain and noise figure of the EDFA used to amplify the comb output and highlights the fact that output line power affects OSNR of the modulated lines. However, OSNR is not the key limiting factor in this experiment, as can be seen from the results for the single laser, which exhibits a similar flattening of the BER curve.
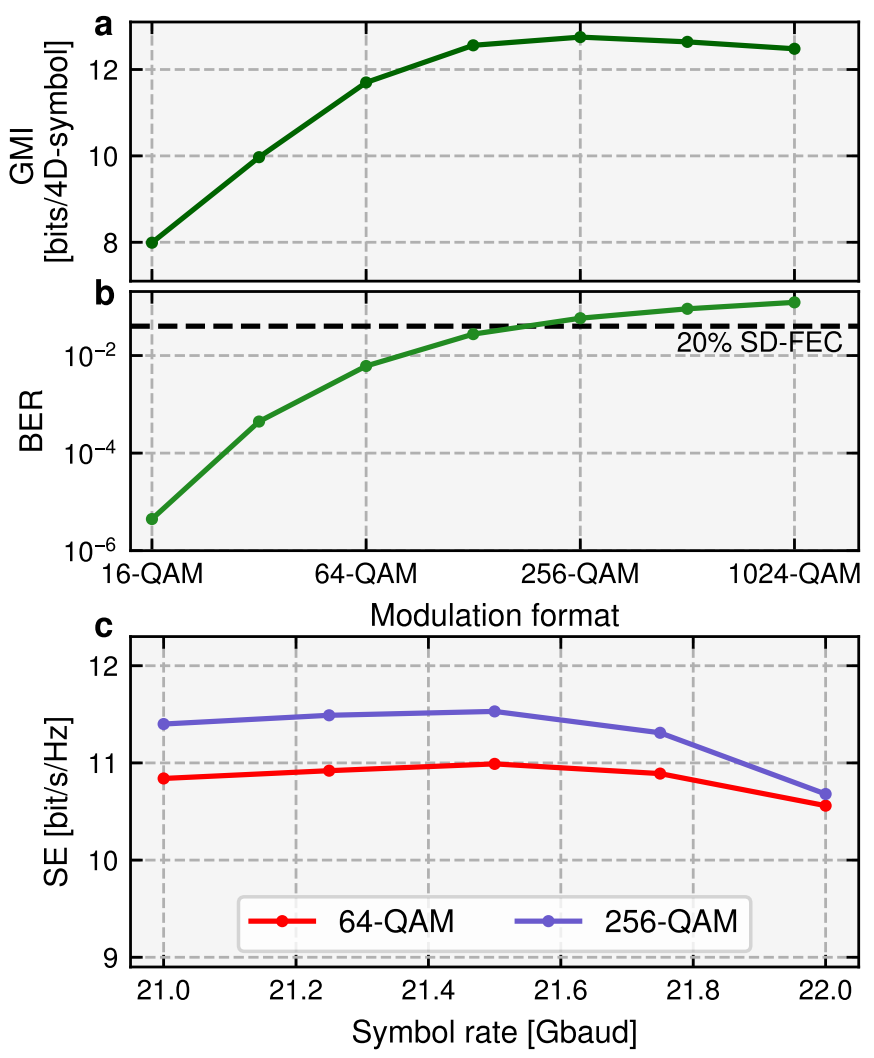

Fig. 5. Back-to-back optimization of modulation formats and symbol rate to maximize the transmitter performance. a single-channel GMI as a function of modulation format for PM-MQAM with $M \in$ $[16,32,64,128,256,512,1024]$. The highest GMI is observed for PM256QAM, reaching about 12.7 bits/4D-symbol. The performance GMI for PM64QAM was about 11.7 bits/4D-symbol. b Corresponding BER as a function of modulation order. With respect to a, we observe that while PM-256QAM results in the highest information rate, it also requires stronger SD-FEC overheads than the typical $20 \%$ indicated in the figure. We therefore choose PM-64QAM as the main modulation format for short-reach (although we also measured using PM-256QAM as a reference). c Optimization of symbol rate to balance overhead from guard-bands and penalty from electrical crosstalk. A symbol rate of $21.5 \mathrm{GBaud}$ on the $22.1 \mathrm{GHz}$ grid provided by the comb resulted in the highest spectral efficiency and was used throughput the transmission experiments. The corresponding overhead for the $600 \mathrm{MHz}$ guard-band was $2.7 \%$.

For 21.5 Gbaud, PM-16QAM we observe an implementation penalty of about $0.5 \mathrm{~dB}$ when using the laser. Using the comb results in a maximum additional penalty of about $0.5 \mathrm{~dB}$, as shown in Fig. 6. We observe the same wavelength dependence as for PM-64QAM but the relative difference is naturally significantly smaller following the lower required OSNR for PM-16QAM.

\section{Single Span}

In a first demonstration we transmitted the superchannel over a single-hop $82 \mathrm{~km}$ standard single-mode fiber (SSMF) link with about $16.5 \mathrm{~dB}$ span loss, as shown in Fig. 7(a). As can be seen performance is very uniform across the whole superchannel with no strong outliers. At optimum launch power the average GMI using PM-64QAM was 10.6 bit/4D symbol corresponding to a SE of $10.1 \mathrm{bits} / \mathrm{s} / \mathrm{Hz}$ after accounting for guard-bands and pilots. This could be slightly increased to 

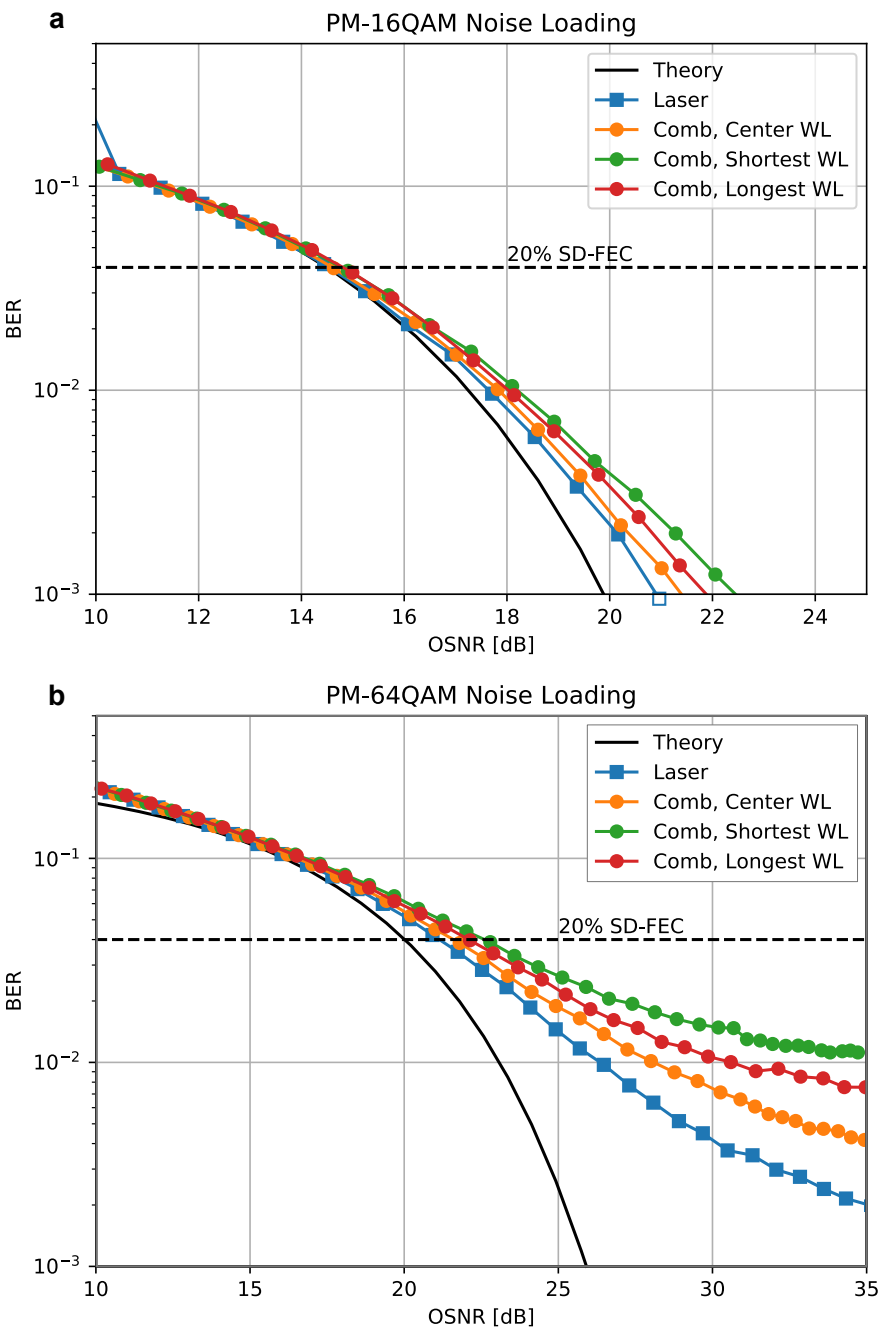

Fig. 6. Noise loading measurements to quantify implementation penalty for a, 21.5 Gbaud PM-16QAM and b 21.5 Gbaud PM-64QAM.

a GMI of $10.9 \mathrm{bit} / 4 \mathrm{D}$ symbol and SE of $10.4 \mathrm{bits} / \mathrm{s} / \mathrm{Hz}$ using PM-256QAM at the expense of increased coding complexity. The corresponding throughput was $11.7 \mathrm{~Tb} / \mathrm{s}(12.0 \mathrm{~Tb} / \mathrm{s})$ for PM-64QAM (PM-256QAM) within a total channel bandwidth of about $1.15 \mathrm{THz}$. Constellation diagrams for three different wavelengths after $82 \mathrm{~km}$ of transmission are shown in Fig. 4b. The PM-16QAM measurements all resulted in a maximum measured GMI of 8 bits/4D-symbol in B2B configuration (Fig. 7(b)), showing that both the throughput and the SE were limited by the modulation format. These single-span results are directly comparable with state-of-the-art bulk comb sources [40].

\section{Long-haul transmission}

We then switched the single span link to a recirculating loop setup to investigate transmission distances reaching $3000 \mathrm{~km}$, focusing on the long-haul transmission aspect of microcombbased superchannels. The average SE, throughput and preFEC BER as a function of transmission distance is shown in Fig. 8. For transmission up to $1500 \mathrm{~km}$ PM-64QAM results in higher SE (and throughput) but comes at the expense of
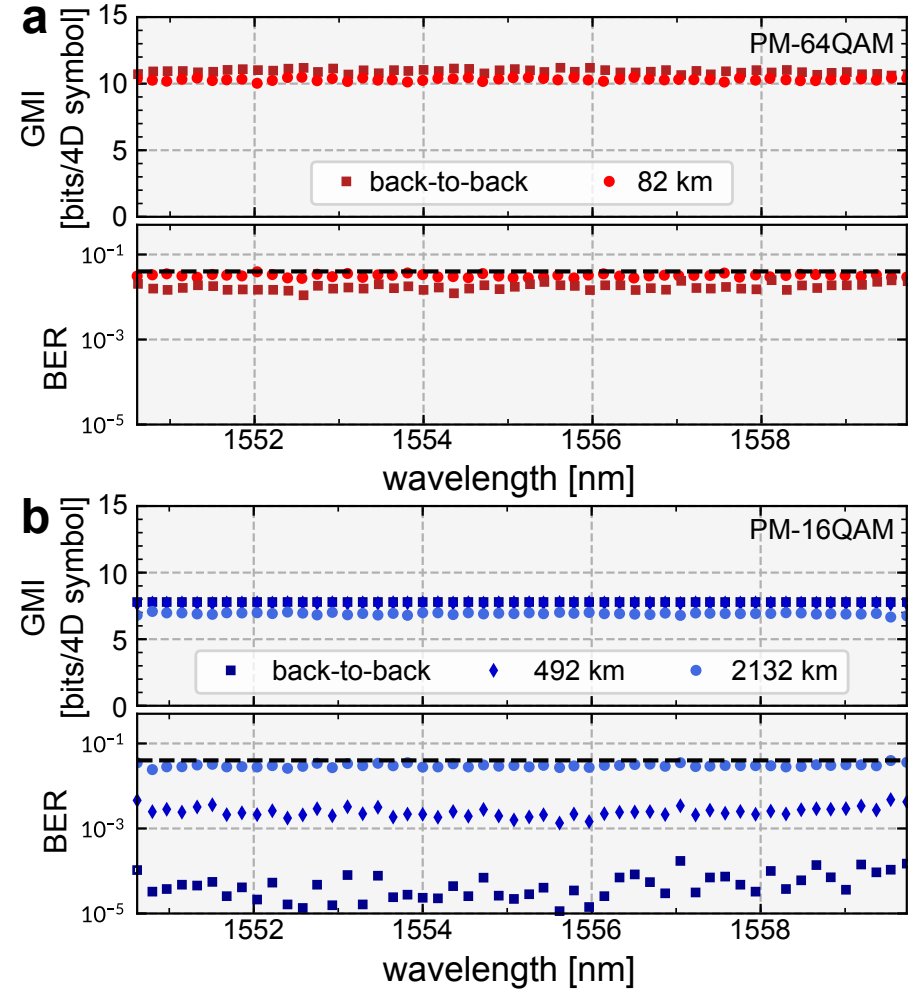

Fig. 7. Transmission results of the superchannel performance per channel for all 52 channels of PM-64QAM (a) and PM-16QAM (b) in terms of bit error rate (BER) and generalized mutual information (GMI). All measurements were done at optimal launch power corresponding to about $13.5 \mathrm{dBm}$ and a symbol rate of $21.5 \mathrm{Gbaud}$. The dashed line indicates the target BER threshold. As can be seen the penalty incurred by modulating the full comb compared to a single channel (Fig. 5(a)) is about $1 \mathrm{bit} / \mathrm{s} / \mathrm{Hz}$.

requiring more powerful FEC, similar to PM-256QAM in the single-span measurement. After $1500 \mathrm{~km}$ the performance is dominated by accumulated amplified spontaneous emission noise and the OSNR is significantly degraded such that PM64QAM does not yield a performance benefit. Note that the reported SE here includes reduction from guard-bands and pilot-overheads, hence the deviation from the theoretical single channel performance of $8 \mathrm{bits} / \mathrm{s} / \mathrm{Hz}$ for short transmission distances in the case of PM-16QAM. Measured performance for each line using PM-16QAM after $2150 \mathrm{~km}$ transmission is shown in Fig. 7. All lines are below the reference threshold [44] for the assumed FEC, verifying that extending transmission beyond $2000 \mathrm{~km}$ is feasible by changing from PM-64QAM to PM-16QAM. At this distance, the SE was $6.7 \mathrm{bits} / \mathrm{s} / \mathrm{Hz}$, corresponding to a throughput of $8.0 \mathrm{~Tb} / \mathrm{s}$. The average superchannel BER for both PM-16QAM and PM64QAM is shown in Fig.(b) 8.

\section{CONCLUSION}

Our results highlight the capability of our microcomb to cope with the various requirements for future transceivers. While the distance covered here spans the range from 80 to $2000 \mathrm{~km}$, even longer distances can be reached by expanding the modulation alphabet with formats such as PM-4QAM and PM-8QAM or probabilistic shaping on constellation formats with higher cardinality [46]. 

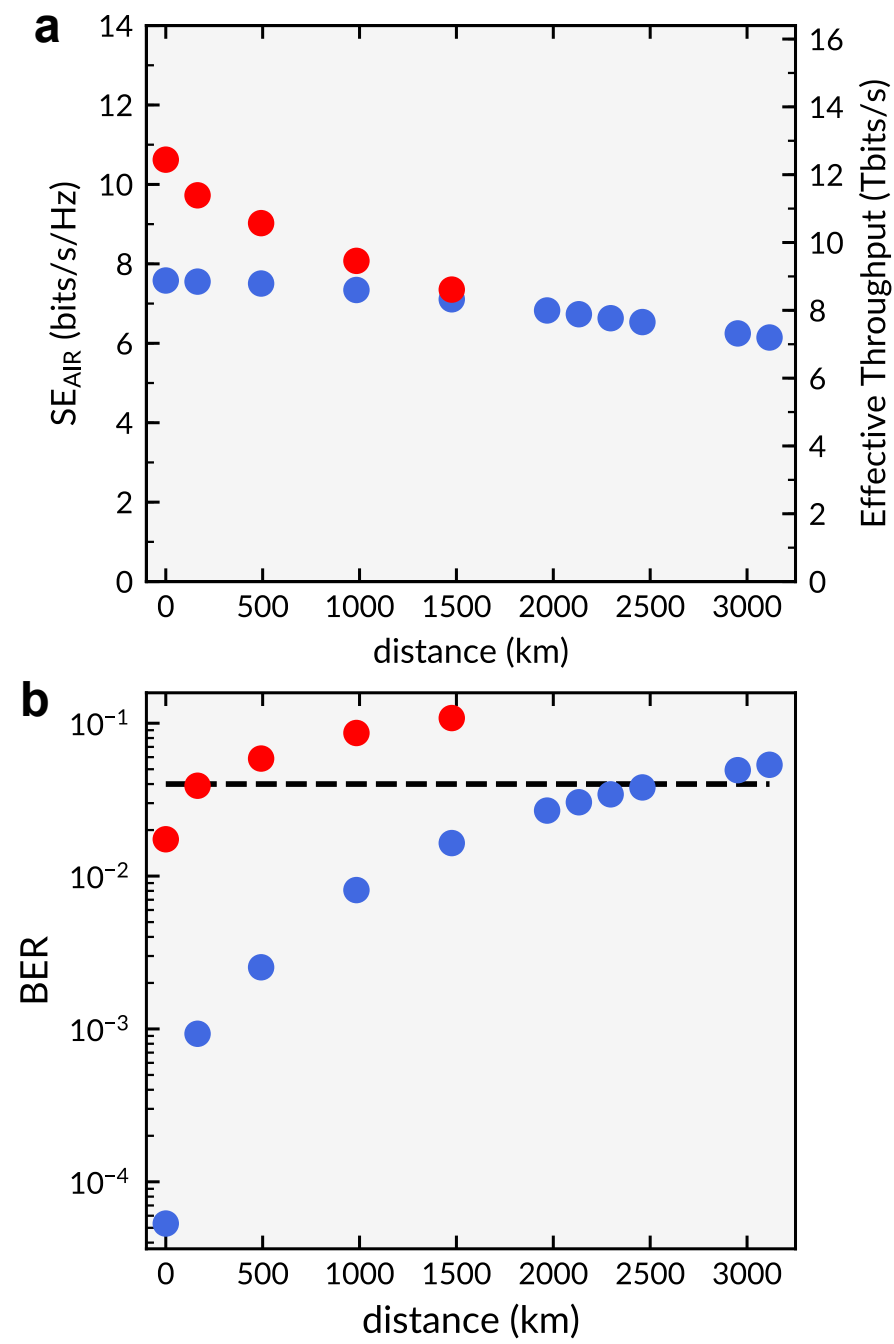

Fig. 8. a Average spectral efficiency and total throughput as a function of transmission distance for PM-64QAM (red) and PM-16QAM (blue). b Average pre-FEC BER as a function of transmission distance.

In summary, we have demonstrated the highest spectral efficiency superchannel transmission using a micro-comb light source operating at a baudrate near its repetition rate and without additional electro-optic generation of frequency components. Combination of the optimal line spacing and the unique stability of soliton microcombs enabled spectral efficiencies exceeding $10 \mathrm{bits} / \mathrm{s} / \mathrm{Hz}$ in a single-span $82 \mathrm{~km}$ link and transmission over distances ranging more than $2000 \mathrm{~km}$ with spectral efficiency exceeding $6 \mathrm{bits} / \mathrm{s} / \mathrm{Hz}$. Rapidly progressing micro-comb research has resulted in micro-combs operating at lower operational power[47], [33], [20], and at much reduced footprint through integration with diode lasers[19], [48] and simplification of microcomb triggering systems[49]. Moreover, the recent advances towards sub-100 $\mathrm{GHz}$ microcombs [50], [51] in SiN resonators and the simultaneous raise in digitalto-analog converter sampling rates [52] promise more wideranging compatibility with transceiver electronics. These advances will greatly reduce the power consumption and form factor of micro-combs, and will eventually allow micro-combbased superchannels to spear-head future highly-flexible alloptical networks requiring multi-Tb/s throughput. Extending high SE systems towards longer transmission lengths will be challenging and likely involve Raman amplification and extremely low loss fibres, however the unique properties of frequency combs which allow WDM nonlinearity compensation [9] could offer significant advantages in these situations.

\section{ACKNOWLEDGEMENTS}

Chalmers acknowledges funding from the Swedish research council (VR) and the European research council (grant 771410). Caltech acknowledges funding from the Air Force Office of Scientific Research (FA9550-18-1-0353) and the Kavli Nanoscience Institute.

\section{REFERENCES}

[1] P. J. Winzer, D. T. Neilson, and A. R. Chraplyvy, "Fiber-optic transmission and networking: the previous 20 and the next 20 years [invited]," Optics Express, vol. 26, no. 18, pp. 24 190-24 239, 2018.

[2] R. W. Going, M. Lauermann, R. Maher, H.-S. Tsai, A. Hosseini, M. Lu, N. Kim, P. Studenkov, S. W. Corzine, J. Summers, M. Anagnosti, M. Montazeri, J. Zhang, B. Behnia, J. Tang, S. Buggaveeti, T. Vallaitis, J. Osenbach, M. Kuntz, X. Xu, K. Croussore, V. Lal, P. Evans, J. T. Rahn, T. Butrie, A. Karanicolas, K.-T. Wu, M. Mitchell, M. Ziari, D. Welch, and F. Kish, "1.00 (0.88) tb/s per wave capable coherent multichannel transmitter (receiver) InP-based PICs with hybrid integrated SiGe electronics," IEEE Journal of Quantum Electronics, vol. 54, no. 4, pp. 1-10, 2018.

[3] G. Liu, K. Zhang, R. Zhang, R. Proietti, H. Lu, and S. J. B. Yoo, "Demonstration of a carrier frequency offset estimator for 16-/32-QAM coherent receivers: a hardware perspective," Optics Express, vol. 26, no. 4, pp. 4853-4862, feb 2018.

[4] J. Rahn, L. Dardis, D. Krause, M. Rice, C. Berry, A. Kumpera, A. Nilsson, X. Xu, K. Croussore, P. Samra, K. Weidner, Z. Morbi, S. DeMars, A. Vasilyev, C. Chen, and P. Freeman, "DSP-enabled frequency locking for near-nyquist spectral efficiency superchannels utilizing integrated photonics," in Proceedings of Optical Fiber Communication Conference, 2018, paper W1B.3.

[5] C. Laperle and M. Osullivan, "Advances in high-speed DACs, ADCs, and DSP for optical coherent transceivers," Journal of Lightwave Technology, vol. 32, no. 4, pp. 629-643, 2014.

[6] S. L. Olsson, J. Cho, S. Chandrasekhar, X. Chen, E. C. Burrows, and P. J. Winzer, "Record-high 17.3-bit/s/Hz spectral efficiency transmission over $50 \mathrm{~km}$ using probabilistically shaped PDM 4096-QAM," in Proceedings of Optical Fiber Communication Conference, 2018, paper. Th4C.5.

[7] M. Mazur, J. Schroeder, A. Lorences-Riesgo, T. Yoshida, M. Karlsson, and P. Andrekson, " $12 \mathrm{bits} / \mathrm{s} / \mathrm{hz}$ spectral efficiency over the c-band based on comb-based superchannels," Journal of Lightwave Technology, vol. 37, no. 2, pp. $411-417,2019$.

[8] F. Klejs, E. P. da Silva, M. Lillieholm, M. P. Yankov, T. Morioka, L. K. Oxenlewe, and M. Galili, "Spectrally Efficient DP-1024QAM 640 Gb/s Long Haul Transmission using a Frequency Comb," in 2020 Optical Fiber Communications Conference and Exhibition (OFC), pp. 1-3.

[9] E. Temprana, E. Myslivets, B. P.-P. Kuo, L. Liu, V. Ataie, N. Alic, and S. Radic, "Overcoming kerr-induced capacity limit in optical fiber transmission," Science, vol. 348, no. 6242, pp. 1445-1448, jun 2015.

[10] M. Mazur, J. Schröder, M. Karlsson, and P. Andrekson, "Joint Superchannel Digital Signal Processing for Effective Inter-Channel Interference Cancellation," Journal of Lightwave Technology, vol. 38, pp. 5676 -5684 .

[11] L. Lundberg, M. Karlsson, A. Lorences-Riesgo, M. Mazur, V. TorresCompany, J. Schröder, and P. Andrekson, "Frequency comb-based WDM transmission systems enabling joint signal processing," Applied Sciences, vol. 8 , no. 5, p. 718, may 2018.

[12] V. Torres-Company, J. Schröder, A. Fulop, M. Mazur, L. Lundberg, O. B. Helgason, M. Karlsson, and P. A. Andrekson, "Laser frequency combs for coherent optical communications," Journal of Lightwave Technology, vol. 37, no. 7, pp. 1663-1670, 2019.

[13] D. T. Spencer, T. Drake, T. C. Briles, J. Stone, L. C. Sinclair, C. Fredrick, Q. Li, D. Westly, B. R. Ilic, A. Bluestone, N. Volet, T. Komljenovic, L. Chang, S. H. Lee, D. Y. Oh, M.-G. Suh, K. Y. Yang, M. H. P. Pfeiffer, T. J. Kippenberg, E. Norberg, L. Theogarajan, K. Vahala, N. R. Newbury, K. Srinivasan, J. E. Bowers, S. A. Diddams, and S. B. Papp, "An optical-frequency synthesizer using integrated photonics," Nature, vol. 557 , no. 7703 , pp. 81-85, 2018. 
[14] M.-G. Suh, Q.-F. Yang, K. Y. Yang, X. Yi, and K. J. Vahala, "Microresonator soliton dual-comb spectroscopy," Science, vol. 354, no. 6312, pp. 600-603, oct 2016.

[15] A. Dutt, C. Joshi, X. Ji, J. Cardenas, Y. Okawachi, K. Luke, A. L. Gaeta, and M. Lipson, "On-chip dual-comb source for spectroscopy," Science Advances, vol. 4, no. 3, p. e1701858, mar 2018

[16] P. Trocha, M. Karpov, D. Ganin, M. H. P. Pfeiffer, A. Kordts, S. Wolf, J. Krockenberger, P. Marin-Palomo, C. Weimann, S. Randel, W. Freude, T. J. Kippenberg, and C. Koos, "Ultrafast optical ranging using microresonator soliton frequency combs," Science, vol. 359, no. 6378, pp. 887-891, feb 2018

[17] M.-G. Suh and K. J. Vahala, "Soliton microcomb range measurement," Science, vol. 359, no. 6378, pp. 884-887, 2018.

[18] Z. L. Newman, V. Maurice, T. Drake, J. R. Stone, T. C. Briles, D. T. Spencer, C. Fredrick, Q. Li, D. Westly, B. R. Ilic, B. Shen, M.-G. Suh, K. Y. Yang, C. Johnson, D. M. S. Johnson, L. Hollberg, K. J. Vahala, K. Srinivasan, S. A. Diddams, J. Kitching, S. B. Papp, and M. T. Hummon, "Architecture for the photonic integration of an optical atomic clock," Optica, vol. 6, no. 5, p. 680, may 2019.

[19] B. Stern, X. Ji, Y. Okawachi, A. L. Gaeta, and M. Lipson, "Batteryoperated integrated frequency comb generator," Nature, vol. 562, pp. 401-405, 2018.

[20] N. Volet, X. Yi, Q.-F. Yang, E. J. Stanton, P. A. Morton, K. Y. Yang, K. J. Vahala, and J. E. Bowers, "Micro-resonator soliton generated directly with a diode laser," Laser \& Photonics Reviews, vol. 12, no. 5, p. 1700307, apr 2018

[21] F. Leo, S. Coen, P. Kockaert, S.-P. Gorza, P. Emplit, and M. Haelterman, "Temporal cavity solitons in one-dimensional kerr media as bits in an all-optical buffer," Nature Photonics, vol. 4, no. 7, pp. 471-476, 2010.

[22] T. Herr, V. Brasch, J. D. Jost, C. Y. Wang, N. M. Kondratiev, M. L. Gorodetsky, and T. J. Kippenberg, "Temporal solitons in optical microresonators," Nature Photonics, vol. 8, no. 2, pp. 145-152, dec 2013.

[23] P. Marin-Palomo, J. N. Kemal, M. Karpov, A. Kordts, J. Pfeifle, M. H. Pfeiffer, P. Trocha, S. Wolf, V. Brasch, M. H. Anderson, R. Rosenberger, V. Kovendhan, W. Freude, T. J. Kippenberg, and C. Koos, "Microresonator-based solitons for massively parallel coherent optical communications," Nature, vol. 546, no. 7657, pp. 274-279, 2017.

[24] Y. Geng, X. Huang, W. Cui, Y. Ling, B. Xu, J. Zhang, X. Yi, B. Wu, S.-W. Huang, K. Qiu, C. W. Wong, and H. Zhou, "Terabit optical ofdm superchannel transmission via coherent carriers of a hybrid chip-scale soliton frequency comb," Opt. Lett., vol. 43, no. 10, pp. 2406-2409, May 2018.

[25] B. Corcoran, M. Tan, X. Xu, A. Boes, J. Wu, T. G. Nguyen, S. T. Chu, B. E. Little, R. Morandotti, A. Mitchell, and D. J. Moss, "Ultra-dense optical data transmission over standard fibre with a single chip source," Nature Communications, vol. 11, no. 1, p. 2568, May 2020.

[26] P. Marin-Palomo, J. N. Kemal, P. Trocha, S. Wolf, K. Merghem, F. Lelarge, A. Ramdane, W. Freude, S. Randel, and C. Koos, "Combbased WDM transmission at $10 \mathrm{Tbit} / \mathrm{s}$ using a DC-driven quantumdash mode-locked laser diode," arXiv e-prints, p. arXiv:1904.11952, Apr 2019.

[27] J. Pfeifle, A. Coillet, R. Henriet, K. Saleh, P. Schindler, C. Weimann, W. Freude, I. V. Balakireva, L. Larger, C. Koos, and Y. K. Chembo, "Optimally coherent kerr combs generated with crystalline whispering gallery mode resonators for ultrahigh capacity fiber communications," Physical Review Letters, vol. 114, no. 9, 2015.

[28] A. Fülöp, M. Mazur, A. Lorences-Riesgo, T. A. Eriksson, P.-H. Wang, Y. Xuan, D. E. Leaird, M. Qi, P. A. Andrekson, A. M. Weiner, and V. Torres-Company, "Long-haul coherent communications using microresonator-based frequency combs," Optics Express, vol. 25, no. 22, pp. 26678-26688, oct 2017

[29] A. Fülöp, M. Mazur, A. Lorences-Riesgo, Ó. B. Helgason, P.-H. Wang, Y. Xuan, D. E. Leaird, M. Qi, P. A. Andrekson, A. M. Weiner, and V. Torres-Company, "High-order coherent communications using modelocked dark-pulse kerr combs from microresonators," Nature Communications, vol. 9, p. 1598, 2018.

[30] H. Lee, T. Chen, J. Li, K. Y. Yang, S. Jeon, O. Painter, and K. J. Vahala, "Chemically etched ultrahigh-Q wedge-resonator on a silicon chip," Nature Photonics, vol. 6, no. 6, pp. 369-373, 2012.

[31] A. H. Atabaki, S. Moazeni, F. Pavanello, H. Gevorgyan, J. Notaros, L. Alloatti, M. T. Wade, C. Sun, S. A. Kruger, H. Meng, K. A Qubaisi, I. Wang, B. Zhang, A. Khilo, C. V. Baiocco, M. A. Popović, V. M. Stojanović, and R. J. Ram, "Integrating photonics with silicon nanoelectronics for the next generation of systems on a chip," Nature, vol. 556, no. 7701, pp. 349-354, apr 2018 .

[32] M. Mazur, M. Suh, A. Fülöp, J. Schröder, V. Torres-Company, M. Karlsson, K. J. Vahala, and P. A. Andrekson, "High spectral efficiency superchannel transmission using a soliton microcomb," in 45th European Conference on Optical Communication (ECOC 2019), 2019, pp. 1-4.

[33] M.-G. Suh and K. Vahala, "Gigahertz-repetition-rate soliton microcombs," Optica, vol. 5, no. 1, pp. 65-66, jan 2018.

[34] X. Yi, Q.-F. Yang, K. Y. Yang, and K. Vahala, "Active capture and stabilization of temporal solitons in microresonators," Optics Letters, vol. 41, no. 9, p. 2037, apr 2016

[35] X. Yi, Q.-F. Yang, K. Y. Yang, M.-G. Suh, and K. Vahala, "Soliton frequency comb at microwave rates in a high-q silica microresonator," Optica, vol. 2, no. 12, pp. 1078-1085, dec 2015.

[36] X. Yi, Q.-F. Yang, K. Y. Yang, and K. Vahala, "Theory and measurement of the soliton self-frequency shift and efficiency in optical microcavities," Optics Letters, vol. 41, no. 15, pp. 3419-3422, 2016.

[37] M. Karpov, H. Guo, A. Kordts, V. Brasch, M. H. Pfeiffer, M. Zervas, M. Geiselmann, and T. J. Kippenberg, "Raman self-frequency shift of dissipative kerr solitons in an optical microresonator," Physical Review Letters, vol. 116, no. 10, p. 103902, 2016.

[38] M.-G. Suh, C. Y. Wang, C. Johnson, and K. J. Vahala, "Directly pumped $10 \mathrm{ghz}$ microcomb modules from low-power diode lasers," Optics letters, vol. 44 , no. 7 , pp. 1841-1843, 2019.

[39] B. Puttnam, R. Luís, W. Klaus, J. Sakaguchi, J.-M. D. Mendinueta, Y. Awaji, N. Wada, Y. Tamura, T. Hayashi, M. Hirano, and M. J., "2.15 $\mathrm{Pb} / \mathrm{s}$ transmission using a 22 core homogeneous single-mode multicore fiber and wideband optical comb," in Proceedings of European Conference on Optical Communication, 2015.

[40] M. Mazur, J. Schröder, A. Lorences-Riesgo, T. Yoshida, M. Karlsson, and P. A. Andrekson, "Overhead-optimization of pilot-based digital signal processing for flexible high spectral efficiency transmission," Optics Express, vol. 27, no. 17, pp. 24654-24669, 2019.

[41] J. Schröder and M. Mazur, "Chalmersphotonicslab/qampy: V0.1," 2018, https://github.com/ChalmersPhotonicsLab/QAMpy.

[42] T. Pfau, S. Hoffmann, and R. Noé, "Hardware-efficient coherent digital receiver concept with feedforward carrier recovery for $M$-QAM constellations," Journal of Lightwave Technology, vol. 27, no. 8, pp. 989-999, 2009.

[43] E. Börjeson, C. Fougstedt, and P. Larsson-Edefors, "ASIC design exploration of phase recovery algorithms for M-QAM fiber-optic systems," in Proceedings of Optical Fiber Communication Conference, 2019, paper W3H.7.

[44] K. Schuh, F. Buchali, W. Idler, T. A. Eriksson, L. Schmalen, W. Templ, R. Schmid, L. Altenhain, M. Moeller, and K. Engenhardt, "Single carrier 1.2 tbit/s transmission over $300 \mathrm{~km}$ with PM-64 QAM at $100 \mathrm{GBaud,"} \mathrm{in}$ Proceedings of Optical Fiber Communication Conference, 2017, paper Th5B.5.

[45] A. Alvarado, E. Agrell, D. Lavery, R. Maher, and P. Bayvel, "Replacing the soft-decision FEC limit paradigm in the design of optical communication systems," Journal of Lightwave Technology, vol. 34, no. 2, pp. 707-721, 2016.

[46] I. F. de Jauregui Ruiz, A. Ghazisaeidi, O. A. Sab, P. Plantady, A. Calsat, S. Dubost, L. Schmalen, V. Letellier, and J. Renaudier, "25.4-Tb/s transmission over transpacific distances using truncated probabilistically shaped PDM-64QAM," Journal of Lightwave Technology, vol. 36, no. 6, pp. 1354-1361, mar 2018.

[47] J. Liu, A. S. Raja, M. Karpov, B. Ghadiani, M. H. P. Pfeiffer, B. Du, N. J. Engelsen, H. Guo, M. Zervas, and T. J. Kippenberg, "Ultralowpower chip-based soliton microcombs for photonic integration," Optica, vol. 5, no. 10, pp. 1347-1353, oct 2018 .

[48] A. S. Raja, A. S. Voloshin, H. Guo, S. E. Agafonova, J. Liu, A. S. Gorodnitskiy, M. Karpov, N. G. Pavlov, E. Lucas, R. R. Galiev, A. E. Shitikov, J. D. Jost, M. L. Gorodetsky, and T. J. Kippenberg, "Electrically pumped photonic integrated soliton microcomb," Nature Communications, vol. 10, no. 1, feb 2019.

[49] N. G. Pavlov, S. Koptyaev, G. V. Lihachev, A. S. Voloshin, A. S. Gorodnitskiy, M. V. Ryabko, S. V. Polonsky, and M. L. Gorodetsky, "Narrow-linewidth lasing and soliton kerr microcombs with ordinary laser diodes," Nature Photonics, vol. 12, no. 11, pp. 694-698, oct 2018.

[50] J. Liu, E. Lucas, A. S. Raja, J. He, J. Riemensberger, R. N. Wang, M. Karpov, H. Guo, R. Bouchand, and T. J. Kippenberg, "Photonic microwave generation in the $\mathrm{X}$ - and $\mathrm{K}$-band using integrated soliton microcombs," Nat. Photonics, vol. 14, no. 8, pp. 486-491, Aug. 2020.

[51] Z. Ye, F. Lei, K. Twayana, M. Girardi, P. A. Andrekson, and V. TorresCompany, in Conference on Lasers and Electro-Optics, p. JTh2F.29.

[52] F. Buchali, V. Lauinger, M. Chagnon, K. Schuh, and V. Aref, "1.1 $\mathrm{tb} / \mathrm{s} / \lambda$ at $9.8 \mathrm{bit} / \mathrm{s} / \mathrm{hz} \mathrm{dwdm}$ transmission over dci distances supported by cmos dacs," in Optical Fiber Communication Conference (OFC) 2020. Optical Society of America, 2020, p. Th3E.2. 